\title{
Papers
}

\section{Effect of bottles, cups, and dummies on breast feeding in preterm infants: a randomised controlled trial}

\author{
Carmel T Collins, Philip Ryan, Caroline A Crowther, Andrew J McPhee, Susan Paterson, Janet E Hiller
}

\begin{abstract}
Objective To determine the effect of artificial teats (bottle and dummy) and cups on breast feeding in preterm infants.

Design Randomised controlled trial.

Setting Two large tertiary hospitals, 54 peripheral hospitals. Participants 319 preterm infants (born at 23-33 weeks' gestation) randomly assigned to one of four groups: cup/no dummy $(\mathrm{n}=89)$, cup/dummy $(\mathrm{n}=72)$, bottle/no dummy $(\mathrm{n}=73)$, bottle/dummy $(\mathrm{n}=85)$. Women with singleton or twin infants $<34$ weeks' gestation who wanted to breastfeed were eligible to participate.

Interventions Cup or bottle feeding occurred when the mother was unable to be present to breast feed. Infants randomised to the dummy groups received a dummy on entry into the trial. Main outcome measures Full breast feeding (compared with partial and none) and any breast feeding (compared with none) on discharge home. Secondary outcomes: prevalence of breast feeding at three and six months after discharge and length of hospital stay.

Results 303 infants (and 278 mothers) were included in the intention to treat analysis. There were no significant differences for any of the study outcomes according to use of a dummy. Infants randomised to cup feeds were more likely to be fully breast fed on discharge home (odds ratio 1.73, 95\% confidence interval 1.04 to $2.88, \mathrm{P}=0.03$ ), but had a longer length of stay (hazard ratio $0.71,0.55$ to $0.92, \mathrm{P}=0.01$ ).

Conclusions Dummies do not affect breast feeding in preterm infants. Cup feeding significantly increases the likelihood that the baby will be fully breast fed at discharge home, but has no effect on any breast feeding and increases the length of hospital stay.
\end{abstract}

\section{Introduction}

Although the benefits of breast feeding preterm infants are well established, practical problems in supporting the transition from tube feeding remain. The most common method of supplementing sucking feeds when the mother is not present is by bottle. This may interfere with breast feeding, possibly because of a difference in sucking action. ${ }^{12}$ An increased prevalence of breast feeding has been reported when bottles were replaced by cups ${ }^{3}$ or tubes. ${ }^{4}$ However, randomised controlled trials provide conflicting evidence on their effect on breast feeding. ${ }^{56}$ While the use of dummies is standard practice for preterm infants and is supported by a reduction in length of hospital stay ${ }^{7}$ their effect on breast feeding is unknown.
We determined the effect of artificial teats (bottle and dummy) and cups on breast feeding in preterm infants $<34$ weeks' gestation at birth.

\section{Methods}

Participants-Women with singleton or twin infants $<34$ weeks' gestation who wanted to breast feed were eligible for inclusion. We excluded infants with congenital abnormalities precluding enteral feeding. Recruitment occurred at two Australian tertiary referral hospitals from April 1996 to November 1999. A plain language information sheet was given to women and written informed consent was obtained from all participants. We included infants requiring transfer to peripheral hospitals, 54 of which participated.

Randomisation and assignment - Women and their infants were randomised to one of four groups: cup/no dummy, cup/dummy, bottle/no dummy, bottle/dummy. An independent researcher developed a separate randomisation schedule for each recruiting hospital by using a random number table to select balanced blocks of varying size with stratification for gestation $(<28$ weeks, 28- $<34$ weeks). Assignments were sealed in sequentially numbered, opaque envelopes. Researchers determined allocation by telephoning an independent ward, available 24 hours a day, within the recruiting hospitals. The mother was the unit of randomisation and twins were assigned to the same group.

Intervention - Cup or bottle feeding commenced at the discretion of the attending nurse/midwife or neonatologist and occurred when the mother was unavailable to breast feed or when additional milk, given orally, was required after a breast feed. Small plastic medicine cups were used as described by Lang. ${ }^{8}$ Infants randomised to the dummy groups had dummies available on trial entry; their use was encouraged during tube feeds and when the infant was restless. For infants who did not receive a dummy, alternate soothing methods were promoted (for example, facilitation of hand to mouth action promoting self quieting behaviour). Recruiting hospitals received education, written instructions, literature, and one-to-one support. Written instructions, literature, and telephone contact were provided to participating peripheral hospitals.

Outcomes-The primary outcomes were the proportion of infants fully breast feeding (compared with partially and not) and the proportion receiving any breast feeding (compared with none) on discharge home. Breast feeding was defined as mother's milk given by direct breast feeding or other feeding device. ${ }^{9}{ }^{10}$ Full breast feeding meant that no other types of milk or solids were given except vitamins and minerals. ${ }^{11}$ Secondary outcomes included the length of hospital stay and prevalence of breast feeding at three and six months after discharge. 


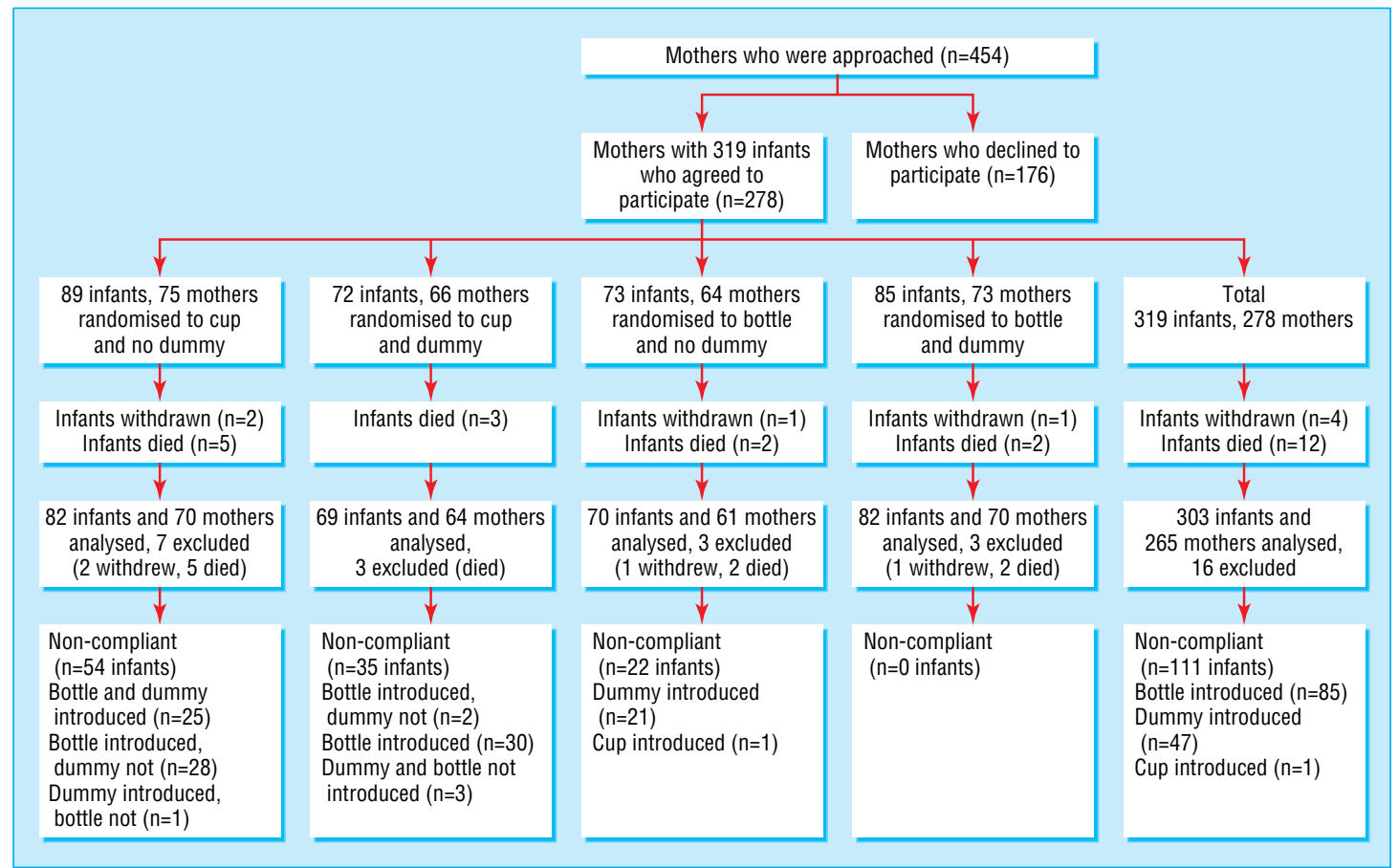

Recruitment and trial participation

Sample size-On the basis of a breast feeding prevalence of $45 \%$ (unpublished hospital data) we calculated that a sample size of 310 could detect a $16.5 \%$ increase in the proportion full breast feeding on discharge home ( $\alpha=0.05,80 \%$ power) between use of dummy use and not (irrespective of cup/bottle) and between cup and bottle (irrespective of use of dummy).

Analysis-All analyses were done on an intention to treat basis. We used logistic regression to estimate odds ratios and 95\% confidence intervals. The number needed to treat and 95\% confidence interval were reported as recommended by Altman. ${ }^{12}$ Kaplan Meier curves and Cox proportional hazards models were used to compare time related variables (days). We accounted for the dependence due to inclusion of twins by using robust variance estimates clustering on the mother. Adjustment was made for analyses where there was a $\geq 10 \%$ difference in distribution and for prespecified factors known to influence duration of breast feeding in preterm infants: maternal education, previous experience of breast feeding, and gestational age. ${ }^{13-18}$

Masking-Participants, care providers, and researchers were not blinded to treatment allocation; data entry and analysis were undertaken unblinded.

Interaction effect-Initial analyses for the primary outcomes showed that there was no clinically important or significant interaction between use of cups and dummies (odds ratio 1.01 (95\% confidence interval 0.36 to $2.79, \mathrm{P}=0.99$ ) for fully versus partially and not breast feeding; $0.94(0.31$ to $2.86, \mathrm{P}=0.91)$ for any versus not breast feeding). On the basis of these results, we performed further comparisons on the marginal groups: cup versus bottle and no dummy versus dummy.

\section{Results}

Participant flow and follow up-We invited 454 women to participate, 176 refused (figure). Reasons for refusal included: wanting to use dummy $(45 / 164,27 \%)$, not wanting to use dummy $(23 / 164,14 \%)$, wanting to use a bottle $(16 / 164,10 \%)$, study did not appeal (36/164, 22\%). Twelve infants died before discharge and four were withdrawn from the study at the mothers' request. Thus, of the 319 infants and 278 mothers enrolled, $303(95 \%)$ and $265(95 \%)$, respectively, were available for the primary analyses (cup/no dummy $n=82$, cup/dummy $n=69$, bottle/no dummy $\mathrm{n}=70$, bottle/dummy $\mathrm{n}=82$ ).

Characteristics of participants-Most maternal and neonatal characteristics were balanced between groups (tables 1 and 2). There was, however, $\mathrm{a} \geq 10 \%$ difference between dummy and no dummy for primiparity and number who had breast fed before and between cup and bottle for primiparity.

Compliance-Non-compliance was high (figure). Of the infants randomised to cup feeding, 56\% (85/151) had a bottle introduced, and of the infants randomised to no dummy $31 \%$ (47/152) had a dummy introduced. Reasons for introducing a bottle were available for $91 \%(77 / 85)$ of the infants, and reasons for introducing a dummy were available for $81 \%(38 / 47)$. For $44 \%(34 / 77)$ the mother decided to introduce a bottle; in $33 \%$ $(25 / 77)$ the decision was taken on the advice of the nurse/midwife (some mothers said both of these had occurred). Of the 77 mothers, 39\% (30) did not like, or had problems with, cup feeding, including the infant not managing cup feeds, spilling a lot, not being satisfied, or taking too long to feed. 12\% $(9 / 77)$ said the staff refused to cup feed their infant. Dummies were introduced because the baby was unsettled $(37 \%, 14 / 38)$ and to teach the baby to suck $(29 \%, 11 / 38)$. Primiparous, tertiary educated women, whose household income was from full time work from either partner, and who had a singleton infant $>28$ weeks' gestation were more likely to have complied with the study protocol.

Breast feeding on discharge home-Not using a dummy had no significant effect on the proportion of infants who were being fully breast fed at discharge $(0.84,0.51$ to $1.39, \mathrm{P}=0.50)$ or partly breast feeding $(0.83,0.45$ to $1.05, \mathrm{P}=0.53)$ (table 3$)$. Cup feeding significantly increased the odds of full breast feeding at discharge (1.73, 1.04 to $2.88, \mathrm{P}=0.03$ ) (table 4 ). The number needed to 
Table 1 Maternal characteristics at trial entry. ${ }^{*}$ Figures are numbers (percentages) unless stated otherwise

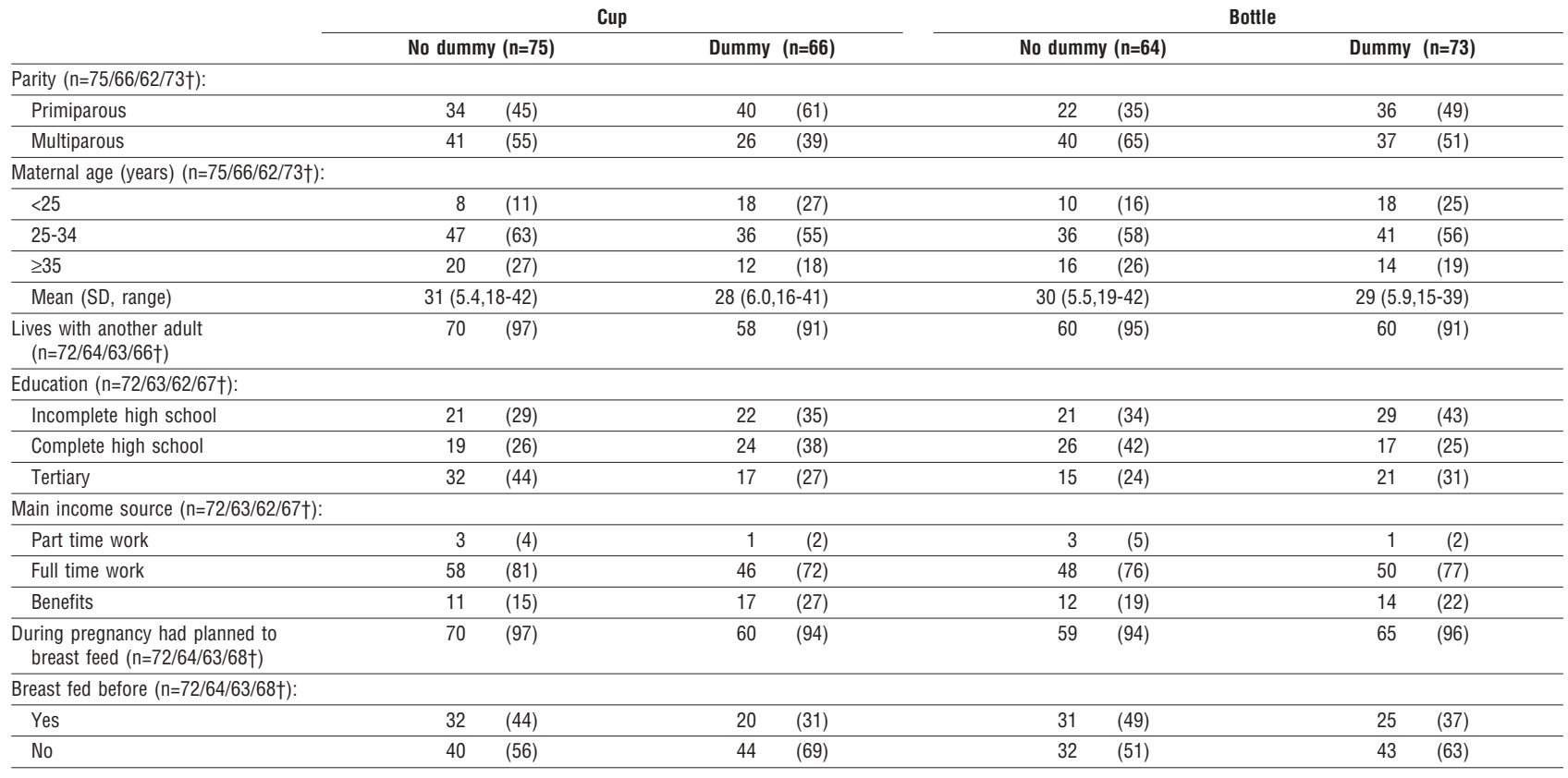

*Percentages may not sum to 100 because of rounding.

†Numerical data available for cup/no dummy, cup/dummy, bottle/no dummy, and bottle/dummy, respectively.

Table 2 Neonatal characteristics at birth. ${ }^{*}$ Figures are numbers (percentages) unless stated otherwise

Cup

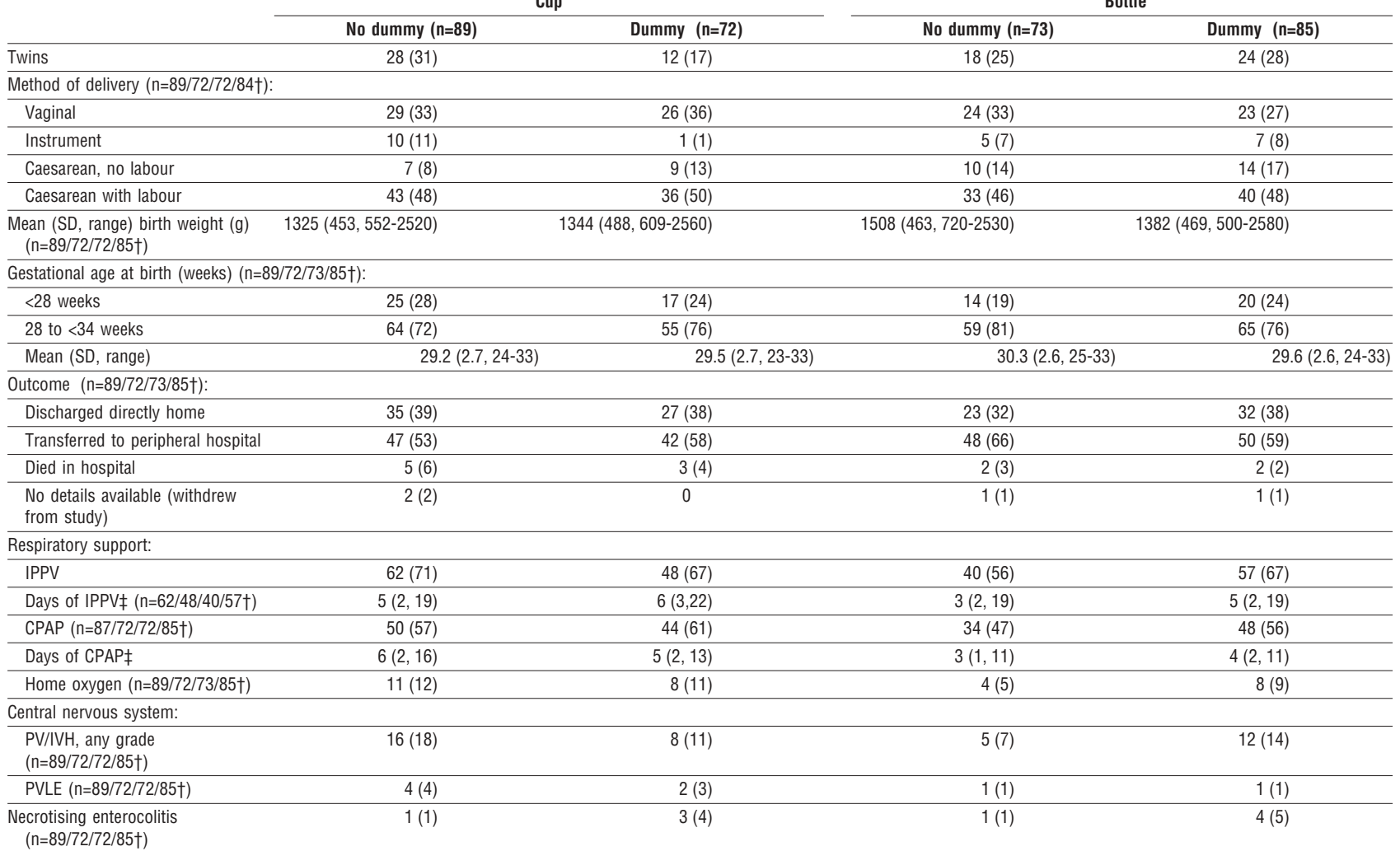

IPPV=intermittent positive pressure ventilation; $C P A P=$ continuous positive airway pressure; $P V / I V H=$ periventricular/intraventricular haemorrhage; PVLE=periventricular leucoencephalopathy.

*Percentages may not sum to 100 because of rounding.

†Numerical data available for cup/no dummy, cup/dummy, bottle/no dummy, and bottle/dummy, respectively

‡Median (25th, 75th centile) 
Table 3 Comparison of prevalence of breast feeding at discharge, 3 months, and 6 months between groups randomised to dummy and no dummy

\begin{tabular}{|c|c|c|c|c|}
\hline & No (\%) with no dummy & No (\%) with dummy & Odds ratio $(95 \% \mathrm{Cl})$; $\mathbf{P}$ value & NNTB*/NNTH† (95\% Cl) \\
\hline \multicolumn{5}{|l|}{ Discharge: } \\
\hline Fully & $79 / 152(52)$ & $85 / 151(56)$ & 0.84 (0.51 to 1.39 ); 0.50 & NNTH 23 (NNTH 6 to $\infty$ to NNTB 15) \\
\hline Any§ & $107 / 152(70)$ & 108/151 (72) & 0.83 (0.45 to 1.50$) ; 0.53$ & NNTH 89 (NNTH 9 to $\infty$ to NNTB 11) \\
\hline $\begin{array}{l}\text { Any breast feeding } 3 \text { months after } \\
\text { discharge§ }\end{array}$ & $58 / 142(41)$ & $53 / 141(38)$ & 0.99 (0.56 to 1.77$) ; 0.98$ & NNTB 31 (NNTH 12 to $\infty$ to NNTB 7 ) \\
\hline $\begin{array}{l}\text { Any breast feeding } 6 \text { months after } \\
\text { discharge§ }\end{array}$ & $43 / 141(30)$ & $34 / 140(24)$ & 1.23 (0.66 to 2.30$) ; 0.51$ & NNTB 16 (NNTH 24 to $\infty$ to NNTB 6) \\
\hline
\end{tabular}

discharge§

${ }^{*}$ No of patients needed to be treated for one additional patient to benefit.

†No of patients needed to be treated for one additional patient to be harmed.

tFully breast fed $v$ combined partially breast fed and not breast fed.

$\S$ Combined fully breast fed and partially breast fed $v$ not breast fed.

treat (where "treatment" means cup feeding) for one extra infant to be discharged home fully breast feeding was seven (95\% CI 4 to 41). Infants randomised to cups were more likely to have any breast feeding, but this was not significant (1.37, 0.78 to 2.38 , $\mathrm{P}=0.27$ ) (table 3). In total 6/265 (2\%) women with 7/303 (2\%) infants chose to express breast milk and bottle feed on discharge home.

Breast feeding at three and six months after discharge-There were no significant differences in the prevalence of any breast feeding in infants randomised to no dummy compared with dummy at three $(0.99,0.56$ to $1.77, \mathrm{P}=0.98)$ and six $(1.23,0.66$ to 2.30 , $\mathrm{P}=0.51$ ) months after discharge (table 3 ). There were minor, non-significant increases in the prevalence of any breast feeding in infants randomised to cup feeds compared with bottle at three (1.31, 0.77 to $2.23, \mathrm{P}=0.33)$ and $\operatorname{six}(1.44,0.81$ to $2.57, \mathrm{P}=0.22)$ months after discharge (table 4).

Length of hospital stay-There was no significant difference (median days, interquartile range) in the length of stay between those randomised to no dummy $(53,35-74)$ or to dummy $(50$, 33-78) (hazard ratio $0.98,0.76$ to $1.26, \mathrm{P}=0.87$ ). Discharge from hospital was significantly delayed for those randomised to cup feeds (cup 59, 37-85; bottle 48, 33-65; 0.71, 0.55 to $0.92, \mathrm{P}=0.01$ ). The differences by gestational age remained significant $(<28$ weeks: cup 93, 86-113; bottle 93, 72-100; $0.55,0.32$ to 0.94 , $\mathrm{P}=0.03 ; 28-<34$ weeks: cup $45,32-66$; bottle $40,32-55 ; 0.69$, 0.52 to $0.93, \mathrm{P}=0.01$ ).

Adverse events -No adverse events were associated with any of the interventions.

\section{Discussion}

The results of our study contribute to the small number of randomised controlled trials that have evaluated the effect of bottle feeding on breast feeding in preterm infants. This study is also the first to evaluate the effect of dummies on breast feeding in this population.

We found no significant differences on any of the study outcomes between those randomised to dummy versus no dummy.
Our study provides no evidence for withholding dummies from infants $<34$ weeks' gestation as a strategy to increase the success of breast feeding. A Cochrane review on the use of dummies in preterm infants showed a significant reduction in hospital stay, ${ }^{7}$ but the two trials included were small. Our trial is the largest to evaluate the effect of dummies and provides evidence that the use of dummies does not reduce length of hospital stay. Infants randomised to cup feeds were significantly more likely to be fully breast fed on discharge home, but also had a longer hospital stay with no significant difference in any breast feeding.

Two previous randomised controlled trials reported conflicting results. ${ }^{5}{ }^{6}$ Kliethermes et al found a significant increase in breast feeding on discharge home when they compared tube feeding $(\mathrm{n}=47)$ with bottle feeding $(\mathrm{n}=52)$ for preterm infants (birth weight 1000-2500 g). ${ }^{5}$ Infants randomised to tube feeds were significantly more likely to be breast feeding (odds ratio 4.5, 1.4 to $15, \mathrm{P}=0.001$, for any breast feeding; $9.4,3.1$ to 28.4 , for full breast feeding). In contrast, Rocha et al found no significant difference in any breast feeding when they compared cup feeding and bottle feeding in infants of 32-36 weeks' gestation (cup 36/44, 82\%; bottle 27/34, 79\%) (full breast feeding was not reported in their study). ${ }^{6}$ Given the strength of the findings of Kliethermes et al, ${ }^{5}$ the significant effect on full breast feeding but not any breast feeding we observed in our study, and the negative findings of Rocha et al, ${ }^{6}$ questions remain as to whether the use of bottles during the transition to breast feeding interferes with a preterm infant's ability to breast feed.

Kliethermes et al found no significant difference in length of stay. ${ }^{5}$ One of the main criteria for discharge home for stable preterm infants is that they can manage all sucking feeds. As the infants matured some became less satisfied with cup feeds and more difficult to feed by this method. The feed was then given by tube, thus delaying the onset of all sucking feeds. As we have few data on this, it is not known how much this may have contributed to the increased length of stay.

Artificial teats may interfere with success of breast feeding because of a difference in sucking action. ${ }^{12}$ We found no difference in breast feeding outcomes with the use of dummies. In the

Table 4 Comparison of prevalence of breast feeding at discharge, 3 months, and 6 months between groups randomised to cup and bottle

\begin{tabular}{|c|c|c|c|c|}
\hline & No (\%) given cup & No $(\%)$ given bottle & Odds ratio ( $95 \% \mathrm{CI}$ ); P value & NNTB*/NNTH† (95\% Cl) \\
\hline \multicolumn{5}{|l|}{ Discharge: } \\
\hline Fully & $92 / 151(61)$ & $72 / 152(47)$ & 1.73 (1.04 to 2.88$) ; 0.03$ & NNTB 7 (4 to 41 ) \\
\hline Any§ & 112/151 (74) & 103/152 (68) & 1.37 (0.78 to 2.38$) ; 0.27$ & NNTB 16 (NNTH 26 to $\infty$ to NNTB 6 ) \\
\hline $\begin{array}{l}\text { Any breast feeding } 3 \text { months after } \\
\text { discharge§ }\end{array}$ & $61 / 144(42)$ & $50 / 139(36)$ & 1.31 (0.77 to 2.23 ); 0.33 & NNTB 16 (NNTH 20 to $\infty$ to NNTB 6) \\
\hline $\begin{array}{l}\text { Any breast feeding } 6 \text { months after } \\
\text { discharge§ }\end{array}$ & 44/142 (31) & $33 / 139(24)$ & 1.44 (0.81 to 2.57$) ; 0.22$ & NNTB 14 (NNTH 32 to $\infty$ to NNTB 6) \\
\hline
\end{tabular}

*No of patients needed to be treated for one additional patient to benefit.

tNo of patients needed to be treated for one additional patient to be harmed.

fFully breast fed $v$ combined partially breast fed and not breast fed.

$\S$ Combined fully breast fed and partially breast fed $v$ not breast fed. 
trial by Kliethermes et al dummies were available for use in both groups, ${ }^{5}$ and it has been shown that infants use the same tongue action (with a different sucking rate) for bottle and dummy teats. ${ }^{1}$ A contributing factor as to why bottles may interfere with success of breast feeding is the immediate and consistent availability of milk with bottle feeding ${ }^{2}$ and not differences in sucking action between breast and bottle or dummy.

\section{Limitations}

A major limitation of our study is the poor compliance, which reduces the power of the trial to identify a real treatment effect. ${ }^{19}$ We are unable to determine if the lack of significant benefit of cup feeding on any breast feeding is due to the low compliance with resultant loss of power, or to lack of efficacy of cup feeding. Similarly, the finding of no benefit attributable to not using a dummy must be qualified by the poor compliance. Exploratory analysis of compliance, however, showed no significant differences in full breast feeding (odds ratio 0.60, 0.26 to 1.37 , $\mathrm{P}=0.22)$, any breastfeeding $(0.75,0.40$ to $1.41, \mathrm{P}=0.37)$, or length of stay (hazard ratio $1.08,0.77$ to $1.51, \mathrm{P}=0.67$ ) with not using a dummy. Compliance analysis also showed a significant increase in the prevalence of any breast feeding with cup feeding (odds ratio 21.09, 2.62 to $169.75, \mathrm{P}=0.004$ ) with no significant difference in length of hospital stay (hazard ratio $0.82,0.58$ to $1.17, \mathrm{P}=0.27$ ). Such compliance analyses need to be interpreted with caution and highlight the need for further research. Compliance differed between recruiting hospitals; the hospital with the better compliance had used cup feeding before, in the other it was introduced for the trial. Most peripheral hospitals had not used cup feeding before. Some staff had strong feelings against cup feeding and the withholding of dummies and some parents did not like cup feeding.

\section{Conclusion}

From our results we cannot support withholding a dummy in preterm infants $<34$ weeks' gestation as a strategy to increase the prevalence of breast feeding. Also, using a dummy does not decrease hospital stay.

The use of cups significantly increased the proportion of infants discharged home fully breast feeding even with the high non-compliance. The obvious benefit of this outcome needs to be considered alongside the financial implications for the health system of longer hospital stays and the lack of effect of cup feeding on any breast feeding.

Given the difficulty of getting staff and parents to accept cup feeding in our trial, the lack of effect on any breast feeding, and the increased length of stay, it is difficult to recommend its introduction. Our study adds some support to the theory that avoiding bottles increases the success of breast feeding, ${ }^{5}$ though more research is needed. Lang ${ }^{20}$ suggests that it may be possible to introduce bottles once breast feeding is well established without interfering with the success of breast feeding. This may be a more acceptable strategy for parents and staff. A sufficiently powered randomised controlled trial is required to test this hypothesis.

We thank the women and their infants and the nursing, midwifery, and medical staff of the following hospitals who participated in the trial: Barmera, Blackwood, Ceduna, Flinders Medical Centre, Gawler Health Service, Kapunda, Lyell McEwin, Modbury, Naracoorte, Port Lincoln, Port Pirie, South Coast District, The Queen Elizabeth, Wallaroo, Western, Women's and Children's (South Australia); Alice Springs, Royal Darwin (Northern Territory); Bairnsdale, Ballarat Base, Bendigo, Box Hill, Central Gippsland Base, Colac Community Health Services, Dandenong, Diamond Valley Community, Frankston, Geelong, Goulburn Valley Base, Hamilton Base, Kilmore and District, La Trobe Regional, Leongatha, Mitcham Private, Monash Medical Centre, Mount Waverley Private, Mt Alexander, Mt Alvernia Mercy, Northern, Northpark Private, Peninsula Private, Sandringham

\section{What is already known on this topic}

The one small randomised controlled trial on the effect of cup feeding on breast feeding in preterm infants showed no benefit

Dummies are widely used for preterm infants but their effect on breast feeding is unknown

\section{What this study adds}

Cup feeding significantly increased the prevalence of fully breast feeding on discharge home but had no effect on any breast feeding and was associated with longer stays in hospital

Use of dummies did not affect breast feeding and did not affect length of stay

and District Memorial, Seymour District Memorial, South Eastern Private, St John of God Health Care, Sunbury Private, Sunshine, The Angliss, The Mercy Hospital for Women, The Valley Private, Wangaratta District Base, Warrnambool and District Base, Werribee Mercy, West Gippsland, Wimmera Base, Wodonga (Victoria). This study was conducted while CTC was a $\mathrm{PhD}$ candidate at the University of Adelaide.

Contributors: CTC initiated the study, formulated the research questions, designed the study, wrote the study protocol and applications for funding, was the principal investigator for the study, undertook recruitment and data collection, entered the data, conducted the data analysis, and drafted the paper. She is guarantor for the paper. PR advised on design and conduct of the study, supervised the statistical analysis, and contributed to the writing of the paper. CAC and AJMcP participated in protocol design and contributed to the interpretation of results and writing of the paper. SP undertook recruitment, participated in data collection, and contributed to the interpretation of results and writing of the paper. JEH contributed to the interpretation of results and writing of the paper. A Moorehead and A Watkins gained the collaboration of the Mercy Hospital for Women and obtained funding from Mercy Hospital for Women Nurses Research Fund. Funding: Mercy Hospital for Women Nurses Research Fund. CTC received a two year midwifery fellowship from the Women's and Children's Hospital Foundation.

Competing interests: None declared.

Ethical approval: Approval was given by the recruiting hospitals research and ethics committees.

1 Bu'Lock F, Woolridge MW, Baum JD. Development of co-ordination of sucking, swallowing and breathing: ultrasound study of term and preterm infants. Dev Med Child Neurol 1990;32:669-78.

2 Neifert M, Lawrence R, Seacat J. Nipple confusion: toward a formal definition.J Pediatr Neifert M, Lawren

Lang S, Lawrence CJ, Orme RL. Cup feeding: an alternative method of infant feeding. Arch Dis Child 1994;71:365-9.

Arch Dis Child 1994;71:365-9.
Stine MJ. Breastfeeding the premature newborn: a protocol without bottles.J Hum Lact Stine MJ. Breastf
1990;6:167-70.

5 Kliethermes PA, Cross ML, Lanese MG, Johnson KM, Simon SD. Transitioning preterm infants with nasogastric tube supplementation: increased likelihood of breastfeeding.J Obstet Gynecol Neonatal Nurs 1999;28:264-73.

6 Rocha NM, Martinez FE, Jorge SM. Cup or bottle for preterm infants: effects on oxygen saturation, weight gain, and breastfeeding. J Hum Lact 2002;18:132-8.

Pinelli J, Symington A. Non-nutritive sucking for promoting physiologic stability and nutrition in preterm infants. Cochrane Database Syst Rev 2004;(4): CD001071.

Lang S. Cup feeding: an alternative method. Midwizes Chromicle 1994;107:171-6.

9 World Health Organization. Indicators for assessing breast-feeding practices. Geneva: WHO, 1991.

10 Hill PD, Hanson KS, Mefford AL. Mothers of low birthweight infants: breastfeeding patterns and problems. J Hum Lact 1994;10:169-76.

11 Labbok M, Krasovec K. Toward consistency in breastfeeding definitions. Stud Fam Plann 1990;21:226-30.

12 Altman DG. Confidence intervals for the number needed to treat. BMJ 1998;317:130912.

13 Kaufman KJ, Hall LA. Influences of the social network on choice and duration of breast-feeding in mothers of preterm infants. Res Nurs Health 1989;12:149-59.

14 Yip E, Lee J, Sheehy Y. Breast-feeding in neonatal intensive care.J Paediatr Child Health 1996;32:296-8.

15 Boo NY, Goh ES. Predictors of breastfeeding in very low birthweight infants at the time of discharge from hospital. J Trop Pediatr 1999;45:195-201.

16 Nyqvist KH, Ewald U. Infant and maternal factors in the development of breastfeeding behaviour and breastfeeding outcome in preterm infants. Acta Paediatr 1999;88:1194203. 
17 Killersreiter B, Grimmer I, Buhrer C, Dudenhausen JW, Obladen M. Early cessation of breast milk feeding in very low birthweight infants. Early Hum Dev 2001;60:193-205.

18 Furman L, Minich N, Hack M. Correlates of lactation in mothers of very low birth weight infants. Pediatrics 2002;109:e57. www.pediatrics.org/cgi/content/full/109/4/ e57 (accessed Sep 2002).

19 Prescott R, Counsell C, Gillespie W, Grant A, Russell I, Kiauka S, et al. Factors that limit he quality, number and progress of randomised controlled trials. Health Technol Assess 1999;3:1-143.

20 Lang S. Breastfeeding special care babies. London: Bailliere Tindall, 1997.

(Accepted 6 May 2004)

doi $10.1136 /$ bmj.38131.675914.55

Department of Nursing and Midwifery Research and Practice Development, Women's and Children's Hospital, 72 King William Road, North Adelaide, South Australia, 5006, Australia

Carmel T Collins research nurse/midwife
Department of Public Health, University of Adelaide, Adelaide, South Australia, 5005, Australia

Philip Ryan associate professor

Janet E Hiller professor

Department of Obstetrics and Gynaecology, University of Adelaide, Adelaide, South Australia, 5005, Australia

Caroline A Crowther professor

Department of Neonatal Medicine, Women's and Children's Hospital, 72 King

William Road, North Adelaide, South Australia, 5006, Australia

Andrew J McPhee deputy head of neonatal medicine

Department of Neonatal Services, Mercy Hospital for Women, Clarendon Street,

East Melbourne, Victoria, 3002

Susan Paterson research midwife

Correspondence to: C Collins collinsct@mail.wch.sa.gov.au 